\title{
User study on remotely controlled UAVs with focus on interfaces and data link quality
}

\author{
Maik Riestock, Frank Engelhardt, Sebastian $\mathrm{Zug}^{1}$ and Nico Hochgeschwender ${ }^{2}$
}

\begin{abstract}
The successes of teleoperation scenarios for mobile robots depends on a stable and reliable communication link. The environment information collected by the robot - represented by 2D or 3D images - has to be provided with a high resolution and a low delay to ensure a fast and precise system response. But in most realistic applications, the communication parameters fluctuate strongly over time. It is necessary to monitor the communication link continuously to react in case of reduced bandwidth and increased delay.

But which environment information and correspondingly which bandwidth is necessary to control a robot safely? Due to a missing reliable rule set we investigated this question for a UAV scenario based on two different environment representations (camera images, gridmaps). We designed a simulator based study and evaluated the capability of the participants to control a robot in case of delayed or coarsely rasterized information. Although our study involved only non-experts, we found some interesting first results. While the performance of our participants correlates strongly with the delay, it is nearly uncorrelated with the image resolution, which suggests downsampling as a valid response for bandwidth decrease. We also found that participants generally struggle with using grid map for controlling the robot. However, this type of interfaces requires far less bandwidth than images. They also excel in situations with higher delays, which makes them the tool of choice when there are really bad channel conditions.
\end{abstract}

\section{INTRODUCTION}

The application of remotely controlled or teleoperated (mobile) robots nowadays range from industrial or military scenarios up to disaster relief and surgical procedures. In industrial scenarios various different techniques or standards exist to ensure and manage the communication link quality, but as the mars rover proves as an extreme and working, parameters such as bandwidth or delay may not that critical as expected. Especially if the system itself is in a safe state without user interaction or if variations in communication quality can be tolerated by reducing the operational speed. However, this is not the case for remotely controlled Unmanned Aerial Vehicles (UAVs). Although the third dimension eases the exploration and accessabilty of various areas, it requires fast and continuous communication. Quadrotors tend

*This work was partially supported by the German Research Foundation (DFG) research project "MoCoRo Plattform für mobile kooperative Robotik"

${ }^{1}$ Maik Riestock, Frank Engelhardt, Sebastian Zug are with Faculty of Computer Science, Otto-von-Guericke University, 39106 Magdeburg, Germany riestock, fengelha, zug@ovgu.de

${ }^{2} \mathrm{Nico}$ Hochgeschwender is with the Department of Computer Science, Bonn-Rhein-Sieg University, Sankt Augustin, Germany nico.hochgeschwender@h-brs.de to be very demanding in teleoperation due to their 6-degreesof-freedom movement, agility and aerodynamic instability.

In the humanitarian disaster aid scenario that we envision (with no line-of-sight), communication bandwidth is a limited and precious good that varies over time, see the work on rescue robotics in [1]. The area itself and its characteristics as well as the distance to the human operator and other external interference sources affect the communication link quality.

Teleoperated robotic scenarios are commonly defined as closed loop control applications. Sensors attached to a mobile robot or manipulator generate input data for the human operator. By interpreting the received data he/she releases commands send back to the robotic entity. Depending on the type of application different visual representations can be exploited, whereby video streams are dominant in most areas. In case of limited bandwidth, video streams have to be downsampled or compressed. Since the rise of $2 \frac{1}{2} \mathrm{D}$ and $3 \mathrm{D}$ sensors and increased computational power also 3D maps get transmitted, with reduced visual complexity and lower bandwidth requirements compared to video streams.

There has been a lot of research in grounded remotely operated robots, but the research in remotely controlled UAVs only started. That is why we investigate the effect of different interfaces (video steam and 3D map) and variable modalities (level of detail and delay) on the operator's performance. If we want to react flexibly on a decreasing bandwidth by reducing the image resolution for instance, we have previously to identify the minimal requirements for an interface representation to ensure a reliable operation mode. Otherwise, it may be applicable to switch it off and to change to another, as it is illustrated in Fig. 1] with different interface representation settings.

Our study is focused on applicability and replicability 1 , both in terms of methodical correctness. On the other hand, we wanted to give a detailed instruction manual for other researchers in that field on how to design and carry out their studies. That is why we show the study design and our process of evaluation, model building and rule generation to draw conclusions regarding the setup. The study design is applicable on other scenarios too. The goal of this approach was to quantify valid interface-communication setups and to provide rules for optimal self-adaptation.

\section{STATE-OF-THE-ART}

The problem of teleoperating UAVs has been widely addressed in literature. Several types of feedback to the

${ }^{1}$ all measured data can be found at https://osf.io/ysbdb/ 

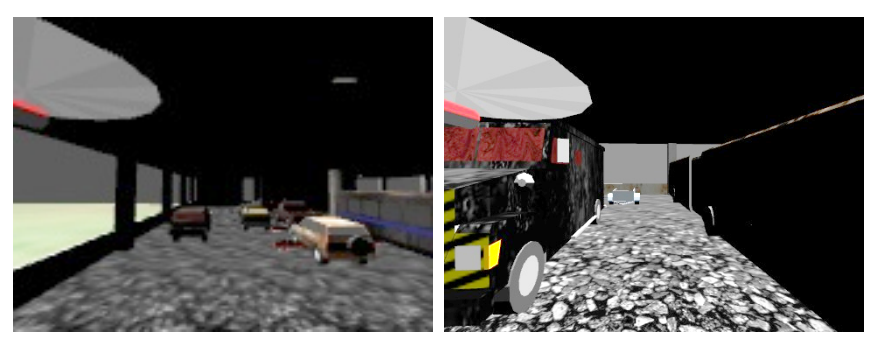

(a) Camera image $160 \times 120$ pixel (b) Camera image $480 \times 320$ pixel $\left(0.91 \mathrm{MB} \mathrm{s}^{-1} @ 20 \mathrm{~Hz}\right)$

(7.48 $\left.\mathrm{MB} \mathrm{s}^{-1} @ 20 \mathrm{~Hz}\right)$

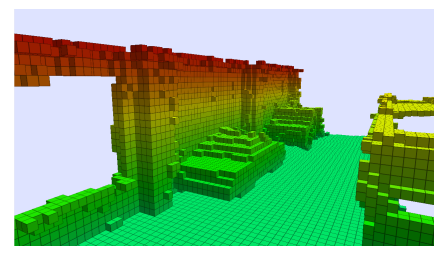

(c) Gridmap voxel length $0.1 \mathrm{~m}$ (d) Gridmap voxel length $0.35 \mathrm{~m}$ (0.91 $\left.\mathrm{MB} \mathrm{s}^{-1} @ 20 \mathrm{~Hz}\right)$

$\left(0.07 \mathrm{MB} \mathrm{s}^{-1} @ 20 \mathrm{~Hz}\right)$

Fig. 1. Different configurations of operator interfaces used in the evaluation of the control performance

operator were investigated, e.g. haptic [2] feedback, camera streams [3], [2], [4], [5] or shared 3D maps [6], [7]. In [2] the authors have built a remote-controlled quadrotor system using only onboard sensors to control the quadrotor and to provide feedback to the operator. In a demonstration, the operator was able to navigate the quadrotor through an office environment without having physical line of sight, but by using only its onboard sensors. In [5] the authors use visual feedback for a simulated helicopter and reduce the effects time-varying delay from the communication channel using a proportional damping injection. In simulation experiments, an operator flies the UAV via an onboard camera. Neither bandwidth consumption of the sensor stream nor the use of mapping information was considered. In [7] the authors use a quadrotor which performs real-time dense 3D reconstruction with the resulting map being synchronized with an operator's workstation. While the mapping process can be viewed in real-time when a stable wireless link is given, the focus of this work is on the highest possible accuracy of the resulting map. No user study was conducted.

The central question that we ask is, what quality is required for the information presented to the operator so that he/she is able to fulfill his/her task. However, several studies [8], [9], [10], [11], [12], [13] have been conducted in general teleoperation scenarios. In these studies, operators perform a given remote-control task while the operator's cognitive load, workload, performance or situation awareness are assessed. None of the studies focuses on the impact of limited bandwidth or delay, thus always assuming a good information quality presented to the operator. Table [ summarizes these studies. They are all from the robotics domain, either driver assistance, urban search and rescue (USAR) or manipulation, with either unmanned air (UAV) or ground vehicles (UGV), robotic arms or cars being involved.
The studies from Horsch et al. [9] and Smets et al. [11] have the closest related setting to ours since they focus on object classification in real environments, however, a stable communication link is assumed between the robot and the operators desk.

\section{SCENARIO DESIGN ANALYSIS AND STUDY DESIGN}

\section{A. Scenario}

In this section we describe the first step of a study design process. Based on an exemplary set of hypotheses we discuss the configuration of the test conceptually.

Fig. 1 illustrates our UAV scenario for evaluating the effect of communication parameters related to the collision probability. Our focus on a safe operation of the UAV rather than a fast mission completion, so effects on the maneuver speed are not considered. For a generic result we assume two interfaces for the pilot, a camera image generated by an onboard camera and a 3D grid map that integrates the current point cloud information in a voxel map. Both approaches differ in terms of required computational performance, usability and most important related to bandwidth.

We formulate the following two exemplary hypothesis:

H1 Operators using a grid map interface has a similar performance than using a camera interface.

$\mathrm{H} 2$ Operators performance suffer less under a change of communication quality using a grid map interface than using a camera interface.

Additionally, we include a set of assumptions and conditions in our design process. We consider the two most important communication parameters as conditions: Communication delay and bandwidth. Hence it is necessary to provide an experimental scenario that allows to control these values independently in a reproducible way.

\section{B. Study Design}

1) Within vs Between Subject Design: In general, the goal of studies is to detect differences between one or more conditions. There are two basic design concepts how to carry out a study, within and between subject design. In withinsubject design, all participants experience all conditions. In contrast, a between-subject design faces the participants with one condition only. The most important disadvantage of this design is caused by the huge amount of participants in order to cover all conditions. The results of a within-subject study are affected by the knowledge of the participant based on previous runs. Other types of effects are the carry over- and position-effects,[14]. They can result from a specific order of conditions or just condition $\mathrm{x}$ before $\mathrm{y}$. These effects can be reduced using control techniques e.g. balanced randomizing the order of conditions and participants.

For our experiment we decide to use a within-subject design concerning the two corresponding challenges, the huge amount of conditions and the powerful learning- effect. A between-subject design would reach it limits with 18 conditions. In order to counteract the learning effect, an extended 
TABLE I

SUMMARY OF OUR PRE-EXAMINED STUDIES

\begin{tabular}{|c|c|c|c|c|c|c|}
\hline Study & Investigation/Task & Domain & Measures & Environment & Autonomy & Participants \\
\hline [8] & $\begin{array}{l}\text { Comparison of display techniques } \\
\text { for assisting in lane changes on a } \\
\text { road }\end{array}$ & $\begin{array}{l}\text { Driver As- } \\
\text { sistance }\end{array}$ & $\begin{array}{l}\text { Attention, } \\
\text { Cognitive load }\end{array}$ & $\begin{array}{l}\text { Realistic simul., dynamic: } \\
3000 \mathrm{~m} \text {, 3-lane, } 60 \mathrm{~km} / \mathrm{h}\end{array}$ & none & $\begin{array}{l}21 \text {, mean age of } 32 \text {, } \\
76 \% \text { male }\end{array}$ \\
\hline [9] & $\begin{array}{l}\text { Comparison of virtual and real } \\
\text { environments: Detect objects and } \\
\text { complete a course }\end{array}$ & $\begin{array}{l}\text { USAR, } \\
\text { UAV }\end{array}$ & $\begin{array}{l}\text { Performance, situa- } \\
\text { tion awareness }\end{array}$ & $\begin{array}{l}\text { artificial simul. and real } \\
\text { world, parkour with obsta- } \\
\text { cles }\end{array}$ & none & $\begin{array}{l}12, \text { mean age of } 25, \\
50 \% \text { male }\end{array}$ \\
\hline$[10]$ & $\begin{array}{l}\text { Design and evaluation of multi- } \\
\text { modal displays. Classify obstacles }\end{array}$ & UGV & Workload & $\begin{array}{l}\text { artificial simul., flat area } \\
\text { with no objects }\end{array}$ & full & 14 , mean age 27 \\
\hline [11] & $\begin{array}{l}\text { Investigation of operator assisting } \\
\text { tools in reconnaissance and victim } \\
\text { search }\end{array}$ & $\begin{array}{l}\text { USAR, } \\
\text { UGV }\end{array}$ & $\begin{array}{l}\text { Performance, } \\
\text { workload, } \\
\text { emotions }\end{array}$ & $\begin{array}{l}\text { real environment, collapsed } \\
\text { tunnel with cars }\end{array}$ & none & $\begin{array}{l}6, \text { mean age } 42, \\
83 \% \text { male, firemen }\end{array}$ \\
\hline [12] & $\begin{array}{l}\text { Difficulty of the ongoing percep- } \\
\text { tion of the relative position and } \\
\text { configuration of a manipulator arm }\end{array}$ & $\begin{array}{l}\text { Robotic } \\
\text { arms }\end{array}$ & $\begin{array}{l}\text { Situation } \\
\text { awareness }\end{array}$ & $\begin{array}{l}\text { realistic simul., exemplary } \\
\text { environment }\end{array}$ & $\underset{\text { partial }}{\text { none }}+$ & $\begin{array}{l}12, \text { mean age of } 23 \text {, } \\
50 \% \text { male, students }\end{array}$ \\
\hline [13] & $\begin{array}{l}\text { Comparison of displays with and } \\
\text { without supporting system for high } \\
\text { speed robots }\end{array}$ & UGV & $\begin{array}{l}\text { Driving } \\
\text { performance }\end{array}$ & $\begin{array}{l}\text { real environment, off-road } \\
\text { course }\end{array}$ & none & 10 , students \\
\hline
\end{tabular}

learning phase was integrated so that every participant were on a similar skill level when starting the experiment.

2) Simulation vs. Real Environment: In a simulation, the UAV, buildings and surrounding including weather and even physics are artificially generated using a software. Whereas, by a real environment, the general approach is to choose or prepare a suitable location and try to keep its integrity for the duration of the experiment. Respectively, both environments provide advantages and disadvantages. While the simulation has easy and comprehensive possibilities of data collection, the real environment is limited by the capability of sensors attached to the robot or surroundings. But also other aspects like controllability and effort regarding location preparation and maintenance can be critical aspects depending on the required level of realism.

The decision was made in favor of the simulation. One aspect is the safety of the application, due to unknown capabilities of the participants we avoid any risks in this way. But more important is the possibility of an comprehensive data collection and to induce artificial communication delay and bandwidth limits to simulate a real link. Additionally, the work of Horsch et al. [9] could not confirm any differences in performance using real or simulated environment.

3) Realistic vs Artificial Scenario: A realistic scenario tries to appear as realistic as possible e.g. by using real objects, whereas the artificial scenario only contain the most necessary elements like walls and foundation without any texture or structure. A realistic scenario provides an evaluation of the hypotheses in an environment close to the intended application within a simulation or a real setup. The highest level of realism can be just reached by field studies.

We prefer to run a realistic scenario in the conditional runs, due to the heavy focus on perception and strong motivation based on rescue missions inside the study. Besides an artificial scenario during the training phase helps to focus on learning the control mechanisms.
4) Steering vs Navigation: The task definition of a remote UAV can cover two roles, the pilot and the mission specialist. Pilot's task is to prepare and maintain the control of the UAV. The mission specialist collects data, operates sensors and advises the pilot where to fly [1]. For practical reasons, we do not reflect this division of labour in our study. A second person would also influence the participant in a nondeterministic way. Our solution is to reduce the navigation effort significantly by designing a single path way that is known to the participants.

\section{STUdy CONCEPT}

The general study concept is structured in two parts. During the first phase a number of studies is executed in order to aggregate all corresponding values necessary to evaluate the hypotheses. Additional information has to be collected to handle the problems coming up with the withinsubject design. The second phase includes the statistical evaluation of the results and the generation of the rule set that gives the adaptations to varying communication parameters.

\section{A. Conditions}

One of our goals is the investigation of the influence of changing level of communication quality on the operators performance, using delay and interface resolution, as described in Sec. III-B.3. Both the grid map and the camera interface work totally different from each other and thus receive a different set of delay and resolution settings. In addition not only the influence of one of those settings needs to be considered but also and more important the combinations of delay and resolution settings. This could easily lead to a combinatorial explosion. For this reason, we limited the number of levels to 3 , resulting in a total number of 18 conditions, 9 for each interface.

Determining appropriate individual settings to investigate is not trivial. The starting point is the definition of the best and worst cases. The definition of the best case is limited 


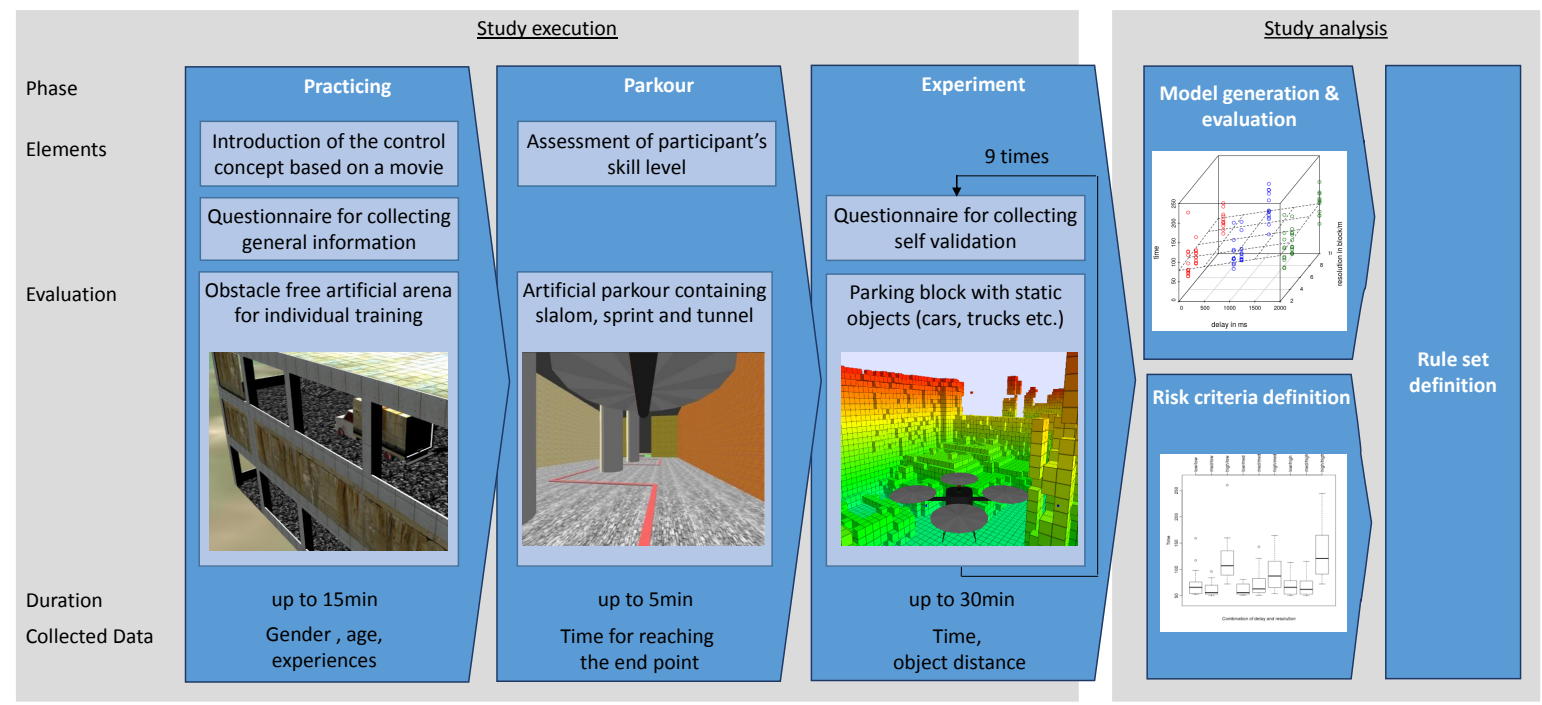

Fig. 2. Structure and key elements of the study

on the network and computational capacity. The worst case was oriented towards the basic conditions a pilot needs to control the UAV. In order to find out, test flights were done using a selection of different settings with high delay and low resolution. Based on the results a selection of settings was made by which it is possible to successfully complete the run. The resulting settings are shown in Tables II III.

The camera resolution is located on the standards used for camera transfers with poor network conditions. For example, video streaming in naval missions using unmanned marine vehicles.[15] The used standards are: QQVGA(160x120), QVGA (320x240) and HVGA(480x320), with 24 bit color resolution each. Grid map resolutions are located below the physical size of the UAV. The size in bytes of the grid map messages is determined by the largest possible point cloud (pcl::PointXYZRGB) needed to transfer the centroids of all occupied grid cells. We assume this to be the case when our Kinect-like sensor model looks at a plain wall at its maximum distance of $5 \mathrm{~m}$. Note that we can map the resolution of both interfaces to the same measure, the message size in $\mathrm{KB}$, giving us the possibility to compare both interfaces regarding bandwidth consumption.

TABLE II

OVERVIEW OF USED SETTINGS (DELAY, RESOLUTION) FOR CAMERA INTERFACE IN DIFFERENT CATEGORIES

\begin{tabular}{|l|c|r||l|l|}
\hline \multicolumn{4}{|c|}{ Camera } \\
\hline \multicolumn{3}{|c|}{ Resolution } & \multicolumn{2}{c|}{ Delay } \\
\hline category & pixel & \multicolumn{1}{|c|}{ data } & category & time \\
\hline low & $160 \times 120$ & $57.6 \mathrm{~KB}$ & low & $0.01 \mathrm{~s}$ \\
mid & $320 \times 240$ & $230.4 \mathrm{~KB}$ & mid & $0.1 \mathrm{~s}$ \\
high & $480 \times 320$ & $460.8 \mathrm{~KB}$ & high & $0.5 \mathrm{~s}$ \\
\hline
\end{tabular}

TABLE III

OVERVIEW OF USED SETTINGS (DELAY, RESOLUTION) FOR GRIDMAP INTERFACE IN DIFFERENT CATEGORIES

\begin{tabular}{|c|c|c|c|c|c|}
\hline \multicolumn{6}{|c|}{ Gridmap } \\
\hline \multicolumn{4}{|c|}{ Resolution } & \multicolumn{2}{|c|}{ Delay } \\
\hline category & voxel size & pixel & data & category & time \\
\hline low & $0.350 \mathrm{~m}$ & $14 \times 10$ & $4.4 \mathrm{~KB}$ & low & $0.1 \mathrm{~s}$ \\
\hline mid & $0.225 \mathrm{~m}$ & $22 \times 15$ & $10.6 \mathrm{~KB}$ & $\operatorname{mid}$ & $1.0 \mathrm{~s}$ \\
\hline high & $0.100 \mathrm{~m}$ & $50 \times 36$ & $57.6 \mathrm{~KB}$ & high & $2.0 \mathrm{~s}$ \\
\hline
\end{tabular}

\section{B. Process}

Every session is separated into three phases and every phase got a specific task and environment. The first phase, the practicing, is necessary because most participants had no experience regarding the remote control of a UAV and thus to counteract the impact of the learning effect. The duration was variable but limited by 20 minutes. The goal was to train every participant to successfully finish the parkour.

The second phase, the parkour, is considered as a test flight. This environment contains a variation of different challenges e.g. slalom, sprint or tunnel. An exercise to ensure that every participant is able to perform the basics movement using a UAV. A successfully finished test-flight was a precondition for the following participation in the experiment.

The third phase, the experiment itself, consist of 9 runs separated by a 1-2 minute break. In each run, another interface setting was used. A car park served as the basis for the environment. The task was to successfully complete the run as fast and fault-free as possible.

\section{Grouping}

First of all, the set of conditions every participant goes through is divided into two subsets. It would take too much time to go through each possible set with each participant, 
which would have resulted in two sessions per participant. This would also reinforce other negative effects. Additionally, already existing effects would be strengthened like fatigue or the learning effect. Due to the split into two sets containing 9 conditions each these problems is avoided.

To spread possible position effects evenly over all conditions, each participant was alternately assigned to one of the two condition sets, whereas every condition was arranged randomly. Every participant was given so many information about the process, UAV, tasks and worlds as possible. This reduces the amount of information gained by each condition and thus reduced their possible influence. For instance, every participant was shown a video at the beginning of the study session. This video contains information about the process of the experiment, basic information about the aircraft, steering of the aircraft. It also shows the simulated environment, explains the task(s) and the goals. Followed by the training phase, which is supposed to reduce the learning effect. A learning curve is characterized by a sharp rise followed by a flattening and thus even a small training can have a big influence on the ability to control a UAV for inexperienced participants. In addition, this reduce person confounding by subjects with more experience by evening out the experience level on all subjects. The last approach was the establishment of a controlled and steady workspace by a strong adherence to the study protocol. The main goal of this approach was to give every participant the same experience during the session and thus reduction of procedural confounding. The following schedule shows the rough version of the study protocol.

TABLE IV

ALL CONDITIONS DIVIDED INTO TWO EVENLY SIZED GROUPS

\begin{tabular}{|l|c|c|c||c|c|c|c|}
\hline \multicolumn{4}{|c||}{ Image } & \multicolumn{4}{c|}{ Gridmap } \\
\hline & \multicolumn{3}{|c||}{ Resolution } & & \multicolumn{3}{c|}{ Resolution } \\
\hline Delay & low & mid & high & Delay & low & mid & high \\
\hline low & 1. & 2. & 1. & low & 2. & 1. & 2. \\
mid & 2. & 1. & 2. & mid & 1. & 2. & 1. \\
high & 1. & 2. & 1. & high & 2. & 1. & 2. \\
\hline
\end{tabular}

\section{Implementation}

The basis of the implementation consists of the operation system Ubuntu and the communication system ROS, Robot Operating System [16]. Based on this comes the simulation software Gazebo,[17]. The UAV and sensors comes from the Hector package,[18]. The Octomap library[19] is used for the generation of grid map, which is then presented using RVIZ. Whereas the software ImgaeView is used for the camerabased interface.

Both simulated environments are build using SketchUp, [20]. The first one, the parkour, is an artificial environment, which served two purposes. The first, as a training area for the participants and thereby it contains multiple challenges like slalom, sprint, and tunnel. The second artificial environment is a car park and serves as realistic scenario, as mentioned in Sec. III-B.3 In contrast to the parkour, here real objects were used as template e.g. cars, trucks, buses.
Additionally, all objects form a passage in order to focus the task more on steering as on navigation, as described in Sec. III-B.4.

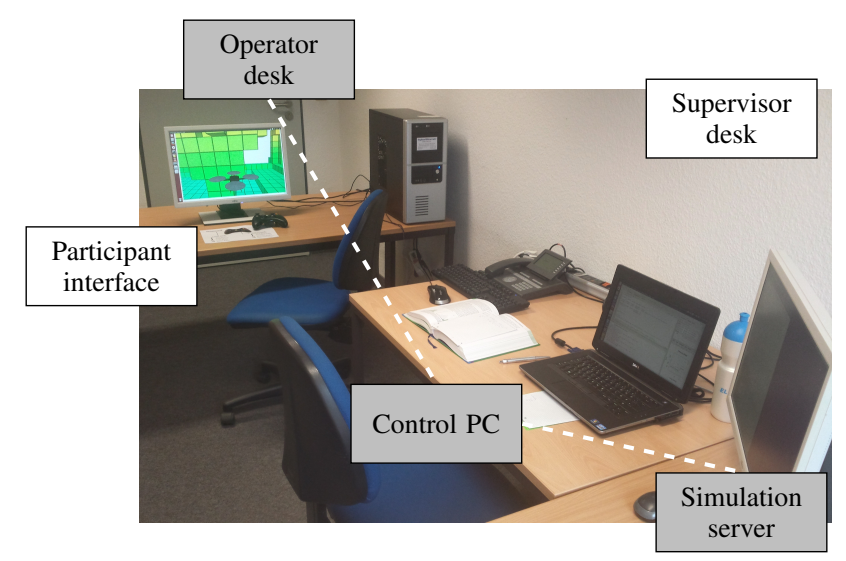

Fig. 3. Study hardware setup combining a simulation server, a pilot desk (with game controller and visual interface) and a supervisor PC

\section{E. Data Aquisition}

The data to collect strongly depends on the goal of the teleoperation, which is in our case the safe navigation to a goal point. The word safe stands for keeping the chance of any collision low during the flight and thus always holding a safe distance to near obstacles. As a numeric value to rate the performance, we measure the distance to near objects. For this purpose we use sensors to measure completion time and distance to near objects inside the simulation.

This distance value is used to calculate the time violating the safety area of the UAV defined by the smallest ellipsoid covering the UAV plus a safety distance (side $0.2 \mathrm{~m}$, top/bottom distance $0.1 \mathrm{~m}$ ).

\section{F. Participants}

31 participants were invited and successfully finished the test-flight. Participants are mostly computer science students or researchers in the field of computer science $(M=$ $24.10, S D=4.86)$.

\section{ANALYSIS}

\section{A. Data Set}

The study produced 279 runs from 31 participants with 9 conditions. 5 Runs were lost due to corrupted data and 46 runs due to crashes. In the end 228 runs could be successfully analyzed. Each data series contains the complete trajectory of the flight, the interface configuration and the participant ID. For each participant we collected personal data and a self-assessment related to the reached performance.

Additionally, due to the splitting in two group of participants as described in Sec. IV-C, a t-test were used in order to determine if there are significant differences between both groups. The resulting $\mathrm{p}$-value $(p=0.2344)$ indicates, that there are no significant differences.

For the exemplary evaluation in this paper we focus on the criticality of a trajectory point based on the distance of 
the UAV to an obstacle as described in Sec. IV-E and in the following sections labeled as collision-time.

\section{B. Model Generation}

The analysis phase of our study focuses on regression analysis, which matches the data on a mathematical representation. The model maps a set of independent variables called predictor, covariable or descriptor variable on a dependent variable [21]. The most common models are linear and linear mixed models. While linear models assume independent data sets, linear mixed models are able to cope with dependent series of data. As described in Sec. III we decided to design a within-subject study, with a sequence of nine runs per participant. Hence, the individual performance of every participates superimposes the results. Consequently, the correlation within the 228 remaining data sets had to be modelled by a more elaborate mixed model.

The statistical significance of the model and thus the fixed effect is indicated by the p-value. This value is calculated using the likelihood ratio test, which compares the two models, one with and one without the fixed effect,[22], [23]. The significance level $\alpha$ was set to 0.05

$$
\begin{aligned}
& \text { collisiontime } \sim \text { delay }+(1 \mid \text { subject }) \\
& \text { collisiontime } \sim \text { delay }+ \text { resolution }+(1 \mid \text { subject })
\end{aligned}
$$

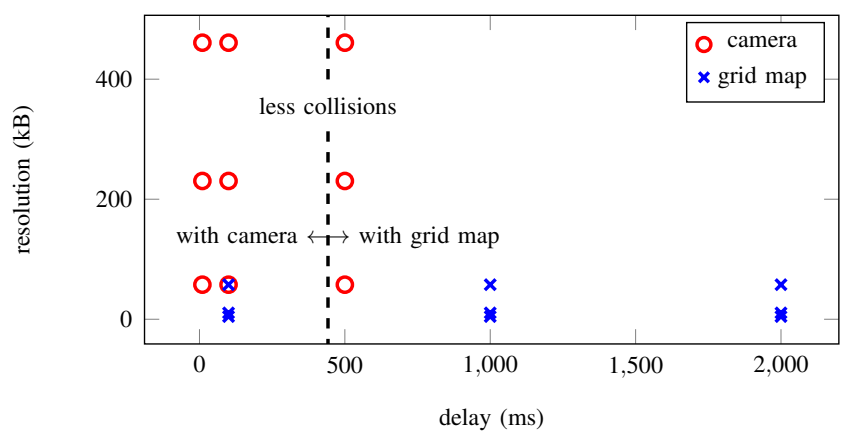

Fig. 4. Scatterplot of the delay and resolution configurations we have investigated in our study. The line $(442 \mathrm{~ms})$ indicates the break-even point of both interfaces regarding the collision probability according to the linear mixed model (Equ. 1b).

\section{Results}

We observed that pilot's had more difficulties keeping a safe distance using the grid map based interface $(M=$ $2.63, S D=3.21)$ as using the camera-based interface $(M=$ $1.33, S D=2.53$ ), the numbers indicates whenever the safety area was violated. These first results speak against our first hypothesis, after which operators using a gridmap interface has a similar performance than using a camera interface.

Coming to the results of the linear mixed models. The results on the camera-based interface indicates that delay $\left(\right.$ coeff $=0.0023496 \pm 0.0004825 \sigma, \chi^{2}(1)=21.654, p=$ $\left.3.265 \times 10^{-6}\right)$ has a significant effect on the collisiontime, increasing the time of an unsafe state of the UAV, whereas resolution $\left(\right.$ coeff $=0.0005002, \chi^{2}(1)=1.771, p=$ $0.1833>0.05)$ has no significant impact. Comparing the results from the camera-based interface to the results of the grid map based interface, we can observe a similar pattern. Here delay $\left(\right.$ coeff $=0.0009508 \pm 0.0003817 \sigma, \chi^{2}(1)=$ $5.7127, p=0.01684$ ) has a significant effect on the collisiontime, increasing the time of an unsafe state of the UAV, whereas resolution $\left(\right.$ coeff $=0.0066577, \chi^{2}(1)=0.0049, p=$ $0.9442>0.05)$ has no significant impact.

The difference between both interfaces lies in the influence of delay indicted by the coefficients coeff $=0.0023496 \pm$ $0.0004825 \sigma$ and coeff $=0.0009508 \pm 0.0003817 \sigma$. Additionally, the $\mathrm{p}$-value of $p=0.01684)$ regarding the gridmap interface is near the edge with the significance level of 0.05 and would fall of with a more strict level. These results indicate that there is a non-trivial level of delay where pilot's using grid map based interface perform better comparing with the camera-based interface. This also favors our second hypothesis, after which a operator performances suffer less under an decrease of communication quality using a grid map interface than using a camera interface.

\section{Risk Criteria Definition}

Based on the findings of the evaluation we assume reduced model only including the delay as fixed effects and the participant as random effect. We make a further step want to know which interface yields best performance under which conditions. We can generate a rule set that indicates when to use which interface to reduce the mission risk. This can be done by bringing both reduced models together, figure 4 . Since both resolution and delay are mapped onto the same respective axis for the two interfaces, the two models appear in the same coordinate space. The result is a intersection line parallel to the resolution axis. But more important is the intersection with the delay axis, stating that if the delay is beyond a threshold the pilot's performance is better using the gridmap interface then using the camera interface. Exemplary, we used the linear models based on collision in order to draw these intersection line. This threshold is uncertain and depends on many factors as the pilots preference and experience, the specific implementation of the interfaces or even the scenario.

\section{CONCLUSION \& FUTURE WORK}

The contribution of this paper is located on two levels. First of all, we described a generic study design for examining the effect of limited communication performance for remote control scenarios. In a second part, the paper is focused on an exemplary study for UAV remote control applications. We investigated two interfaces for UAV pilots and simulated a limited communication bandwidth.

The results showed that the participants had difficulties fulfilling their task using the grid map based interface and thus reject our first hypothesis.

Based on the observation of the sessions and the statement from the participants the mostly mentioned cause for a collision was the presence of visual bugs, e.g. obviously missing blocks or suddenly arising blocks. But also to mentioned is that if participants had experience with remote controlling 
a drone or any robot, no one ever experience a grid map interface. This means, that some difficulties using a grid map originate from the fact that people are unfamiliar with the concept of an artificial interface. These problems need to be addressed in further investigations e.g. gain operators confidence by additionally training. But on the other hand, the results also favored our second hypothesis, where we stated that the operator performances suffer less under a change of communication quality using a grid map interface than using a camera interface. This results lead us to the determination of a risk criteria, which can be used as initial point of a adaptive interface combining both modalities.

The future steps will address the conceptual level as well as the specific study too. Related to the study design we evaluate the remaining data of the experiments in order to generate a set of variables that have to be investigated in general. Additionally, it is intended to repeat the study with professional drone pilots. We expect to prove that a homogeneous skill level simplifies the examination effort.

Focused on the specific scenario we will run an advanced study to confirm the evaluation results and to switch from a simulation based approach to a real environment. The presented work is a key ingredient towards the design and development of self-adaptive interfaces which decide at run time, based on the current communication channel status, which modality in which configuration is presented to the pilot.

\section{REFERENCES}

[1] R. R. Murphy, Disaster Robotics. The MIT Press, 2014.

[2] P. Stegagno, M. Basile, H. H. Bülthoff, and A. Franchi, "A semiautonomous UAV platform for indoor remote operation with visual and haptic feedback," in 2014 IEEE Int. Conf. on Robotics and Automation, Hong Kong, China, 05/2014 2014.

[3] S. Stramigioli, R. Mahony, and P. Corke, "A novel approach to haptic tele-operation of aerial robot vehicles," in Robotics and Automation (ICRA), 2010 IEEE International Conference on, May 2010, pp. 53025308.

[4] T. M. Lam, H. W. Boschloo, M. Mulder, and M. M. van Paassen, "Artificial force field for haptic feedback in uav teleoperation," IEEE Transactions on Systems, Man, and Cybernetics - Part A: Systems and Humans, vol. 39, no. 6, pp. 1316-1330, Nov 2009.

[5] L. R. Salinas, E. Slawiñski, and V. A. Mut, "Complete bilateral teleoperation system for a rotorcraft uav with time-varying delay," Mathematical Problems in Engineering, vol. 2015, 2015.

[6] D. Droeschel, M. Nieuwenhuisen, M. Beul, D. Holz, J. Stückler, and S. Behnke, "Multilayered mapping and navigation for autonomous micro aerial vehicles," Journal of Field Robotics, 2015.

[7] M. Faessler, F. Fontana, C. Forster, E. Mueggler, M. Pizzoli, and D. Scaramuzza, "Autonomous, vision-based flight and live dense $3 \mathrm{~d}$ mapping with a quadrotor micro aerial vehicle," Journal of Field Robotics, vol. 1, 2015.

[8] V. C. Knott, S. Demmelmair, and K. Bengler, "Distraction and Driving Behavior by Presenting Information on an "Emissive Projection Display" Compared to a Head-up Display," Proceedings of the 12th International Conference Engineering Psychology and Cognitive Ergonomics, 2015.

[9] C. H. Horsch, N. J. J. M. Smets, M. A. Neerincx, and R. H. Cuijpers, "Comparing performance and situation awareness in USAR unit tasks in a virtual and real environmen," Proceedings of the 10th International ISCRAM Conference, 2013.

[10] C. M. S. Boris Trouvain, "A Comparative Study of Multimodal Displays for Multirobot Supervisory Control," Proceedings of the 7th International Conference Engineering Psychology and Cognitive Ergonomics, 2007.
[11] N. J. J. M. Smets, M. A. Neerincx, and R. Looije, "Measuring User Behavior in a Complex USAR Team Evaluation," Proceedings of the 8th International Conference on Methods and Techniques in Behavioral Research, 2012.

[12] Y. Fu, W. Chen, S. Li, and Z. Jiao, "Evaluating Operator Performance in Teleoperated Manipulator System Factored by Camera Configurations," Proceedings of the 12th International Conference Engineering Psychology and Cognitive Ergonomics, 2015.

[13] A. Kelly, N. Chan, H. Herman, and R. Warner, "Experimental Validation of Operator Aids for High Speed Vehicle Teleoperation," Proceedings of the 13th International Symposium on Experimental Robotics, 2013.

[14] J. Bortz, Statistik für Human- und SOzialwissenschaftler, C. Schuster, Ed. Springer-Verlag, 2010.

[15] J.-M. Moreno-Roldán, M.-Á. Luque-Nieto, J. Poncela, V. Díaz-del Río, and P. Otero, "Subjective Quality Assessment of Underwater Video for Scientific Applications," Sensors, vol. 15, no. 12, pp. $31723-$ $31737,2015$.

[16] M. Quigley, K. Conley, B. P. Gerkey, J. Faust, T. Foote, J. Leibs, R. Wheeler, and A. Y. Ng, "Ros: an open-source robot operating system," in ICRA Workshop on Open Source Software, 2009.

[17] “Gazebo. Robot simulation made easy." http://gazebosim.org/tutorials? cat=guided_b\&tut=guided_b1, 2016, online; accessed 25-April-2016.

[18] J. Meyer, A. Sendobry, S. Kohlbrecher, U. Klingauf, and O. von Stryk, "Comprehensive Simulation of Quadrotor UAVs using ROS and Gazebo," in 3rd Int. Conf. on Simulation, Modeling and Programming for Autonomous Robots (SIMPAR), 2012, p. to appear.

[19] A. Hornung, K. M. Wurm, M. Bennewitz, C. Stachniss, and W. Burgard, "OctoMap: An efficient probabilistic 3D mapping framework based on octrees," Autonomous Robots, vol. 34, no. 3, pp. 189-206, 2013.

[20] "SketchUp." https://www.sketchup.com, 2016, online; accessed 25April-2016.

[21] F. E. Harrell, Regression Modeling Strategies: With Applications to Linear Models, Logistic Regression, and Survival Analysis. Springer, 2001.

[22] B. Winter, "Linear models and linear mixed effects models in R with linguistic applications," CoRR, vol. abs/1308.5499, 2013. [Online]. Available: http://arxiv.org/abs/1308.5499;http://dblp. uni-trier.de/rec/bib/journals/corr/Winter13

[23] B. M. Bolker, M. E. Brooks, C. J. Clark, S. W. Geange, J. R. Poulsen, M. H. H. Stevens, and J.-S. S. White, "Generalized linear mixed models: a practical guide for ecology and evolution," Trends in ecology \& evolution, vol. 24, no. 3, pp. 127-135, 2009. 